\title{
Analysis Code for High Gradient Dielectric Insulator Surface Breakdown
}

\author{
Phase I Final Report
}

\author{
Grant Number DE-SC0002031
}

Calabazas Creek Research, Inc. 690 Port Drive

San Mateo, CA 94404

(650) 312-9575, Fax: (650) 312-9536

RLI@CalCreek.com

\section{Principal Investigator: Dr. R. Lawrence Ives}

May 30, 2010

These SBIR/STTR data are furnished with SBIR/STTR rights under Grant No. DESC0002031. For a period of four (4) years after acceptance of all items to be delivered under this grant, the Government agrees to use these data for Government purposes only, and they shall not be disclosed outside the Government (including disclosure for procurement purposes) during such period without permission of the grantee, except that, subject to the foregoing use and disclosure prohibitions, such data may be disclosed for use by support contractors. After the aforesaid four-year period, the Government has a royalty-free license to use, and to authorize others to use on its behalf, these data for Government purposes, but is relieved of all disclosure prohibitions and assumes no liability for unauthorized use of these data by third parties. This Notice shall be affixed to any reproductions of these data in whole or in part. 


\section{Introduction}

High voltage (HV) insulators are critical components in high-energy, accelerator and pulsed power systems that drive diverse applications in the national security, nuclear weapons science, defense and industrial arenas. In these systems, the insulator may separate vacuum/non-vacuum regions or conductors with high electrical field gradients. These insulators will often fail at electric fields over an order of magnitude lower than their intrinsic dielectric strength due to flashover at the dielectric interface. Decades of studies have produced a wealth of information on fundamental processes and mechanisms important for flashover initiation, but only for relatively simple insulator configurations in controlled environments. Accelerator and pulsed power system designers are faced with applying the fundamental knowledge to complex, operational devices with escalating HV requirements. Designers are forced to rely on "best practices" and expensive prototype testing, providing boundaries for successful operation. However, the safety margin is difficult to estimate, and system design must be very conservative for situations where testing is not practicable, or replacement of failed parts is disruptive or expensive.

Surface breakdown of insulators poses a challenge across a wide portfolio of Department of Energy (DOE) supported activities; here we will discuss two examplars on which local collaboration is possible to illustrate the issues, but the results can be generalized. Two important pulsed power driven projects within the Science and Technology Principal Directorate at the Lawrence Livermore National Laboratory (LLNL) that are impacted by insulator design are an explosively driven magnetic flux compressor and a compact, high gradient induction accelerator. The magnetic flux compressor is used to generate extreme currents and magnetic fields. A dielectric insulator is located in the power flow section to establish a vacuum region for the experiments. The insulator environment during operation cannot be duplicated in a controlled laboratory test, so the designers use scaled tests and extrapolate. Even this approach is limited due to the lack of basic understanding of how the performance scales to the harsh, complex operational environment. An example of a large scale stacked insulator is shown in Figure 1 [Houck2008b].

Induction electron accelerators are used in many applications, from basic physics to flash radiography. A dielectric insulator separates the source of the accelerating power from the evacuated beam line. Conventional designs locate the insulator farther from the location of the electron beam, sacrificing efficiency and compactness. Novel designs are avoided as testing in an operational environment cannot be accomplished until a significant fraction of the accelerator is assembled. If the design proves unsatisfactory, corrections are prohibitively expensive. Presently, pulsed power system designers lack the computational tools to develop the next generation of systems. An example of an induction accelerator cell vacuum gap is shown in Figure 2 [Houck2008b]. 


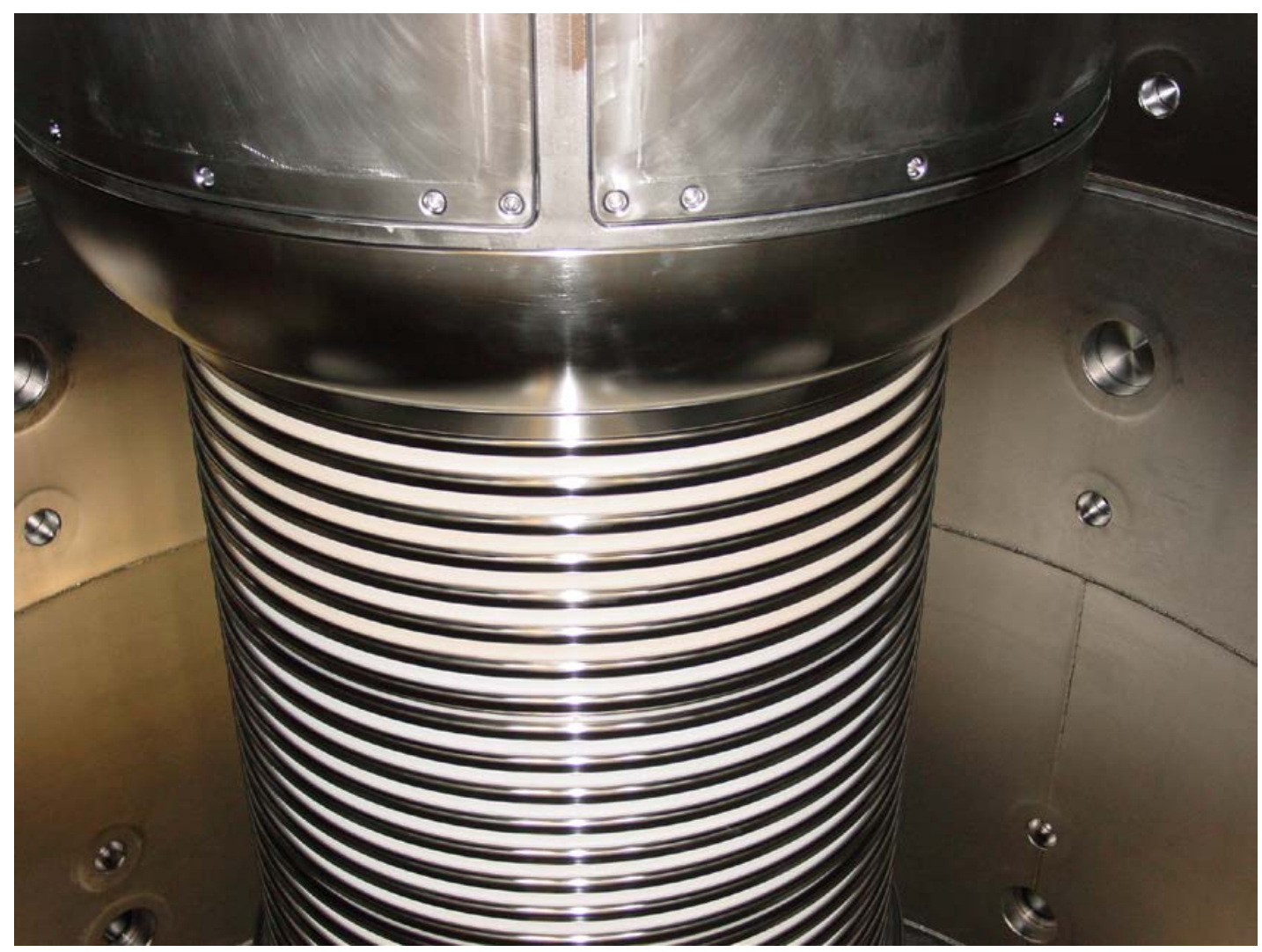

Figure 1. DARHT-2 Injector Column at Los Alamos National Laboratory. The metal-insulator stack is about $3 \mathrm{~m}$ in height, and $1 \mathrm{~m}$ in diameter.

Fundamentally, flashover can occur via dielectric multipactor along the surface of an insulator. Electrons impacting the dielectric surface with energy between $\sim 100 \mathrm{~V}$ and $\sim 1$ $\mathrm{kV}$ can generate emission of multiple secondary electrons, increased for grazing impact. Since the emission energy of secondary electrons is below $100 \mathrm{~V}$, a multipactor discharge only grows when there is an energy source for the electrons, such as an external electric field at least partially aligned with the dielectric. Emission of secondary electrons from the dielectric surface leaves the surface positively charged, and this positive charge affects the electric field such that electrons are attracted back to the surface. This positive feedback effect leads to a rapidly growing electron cloud. The discharge saturates when the field returning the electrons to the surface becomes sufficiently strong that the work done by the tangential electric field decreases due to the shorter electron flight time. Additional sources of electrons in breakdown phenomena include ionization of ambient gas atoms by energetic electrons, thermionic and field emission of electrons from surfaces, surface emission stimulated by UV emitted by excited gas atoms, and ionization and surface emission generated by xrays emitted by the impact of energetic electrons on surfaces. Indeed, electronegative background gases such as oxygen and $\mathrm{SF}_{6}$ can also serve as a sink for electrons through electron attachment processes that form negative ions. 


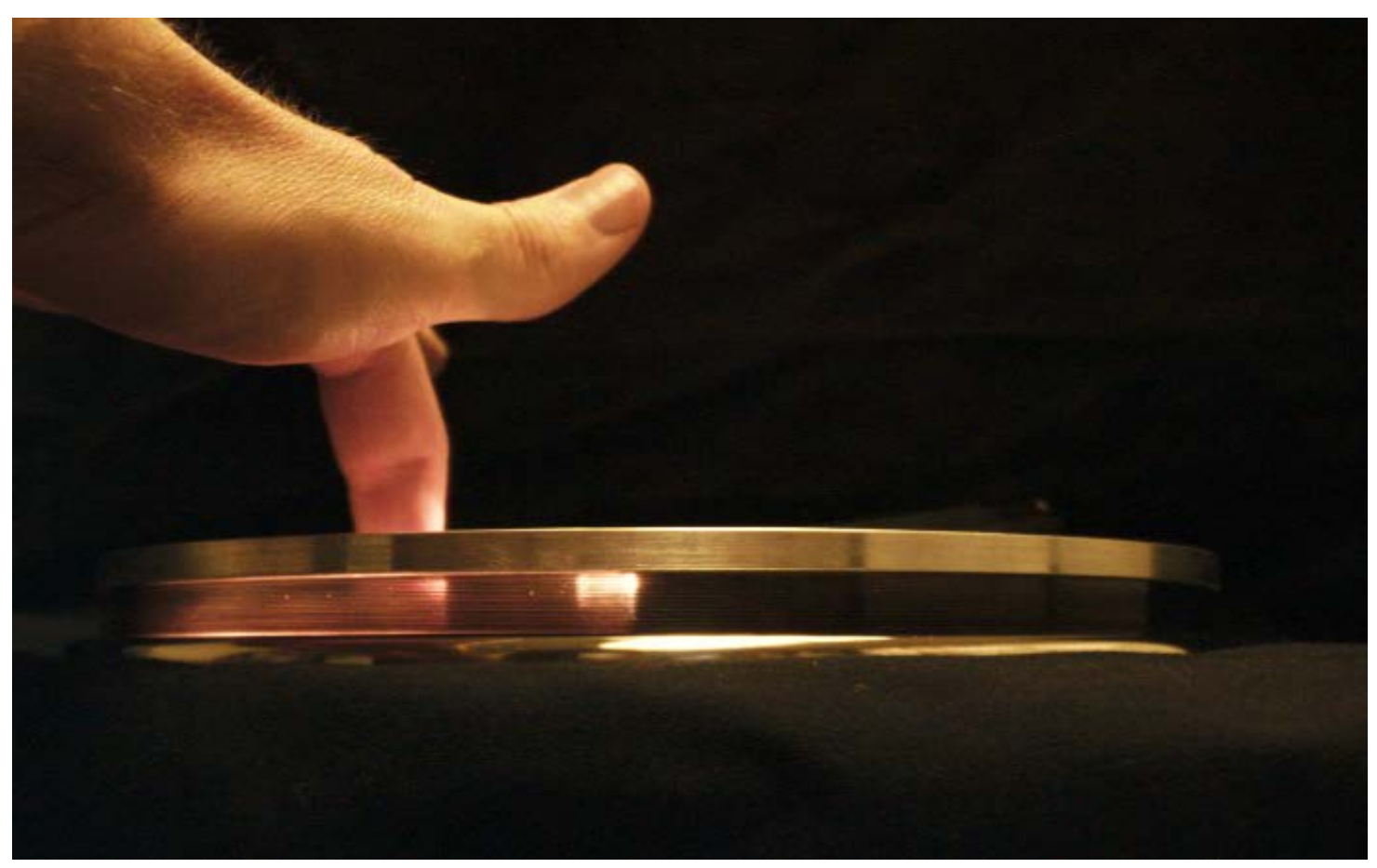

Figure 2. Vacuum gap of an induction accelerator cell.

The myriad electron sources depend on many complicated factors. Material properties govern the energy and angular dependence of secondary emission as well as the dielectric permittivity which distorts the electric field lines between the high voltage conductors. Geometric structure determines the direction of the fields with respect to the dielectric surface and, hence, determines how work is done on the electrons. Surface roughness and microstructure determines local field enhancement critical for determining field emission. Conditioning of the materials determines adsorbed gases and impurities. Driving voltages and frequencies control the electric field strength and direction and its interaction with the electrons. Space charge effects influence the forces on charged particles. Local particle fluxes and surface currents can lead to heating for gas desorption and thermionic emission, as well as generation of xrays. Ambient and desorbed gas constituents determine the atomic collisions and chemistry that influence the electron growth rate. All these processes lead to a highly kinetic system in which the electron energy distribution function (EEDF) can become highly non-Maxwellian.

While simple analysis has successfully determined some trends, for design of mission critical high voltage systems, a comprehensive quantitative treatment is needed. Modeling and simulation can handle the analytically intractable interactions between all the physics described, and perform deeper analysis than is possible in the high voltage and often destructive environment that occurs in breakdown events.

Early efforts [Vogtlin1989] emphasized vacuum insulators related to electron induction accelerators [Scarpetti1997]. This work involved conventional insulator designs and pulses of few to 100's of ns. About 15 years ago a novel insulator design, referred to as a High Gradient Insulator (HGI) [Elizondo1992], was suggested for accelerator applications[Sampayan1995, Houck1997]. The theory of single surface multipactor, 
including a susceptibility diagram, was developed using a Monte Carlo scheme [Kishek1998] and later demonstrated that saturation depends on surface charging rather than beam loading [Ang1998]. The susceptibility diagram was generalized to include the effects of external and RF magnetic fields [Valfells2000a]. Substantial laboratory testing of the HGI continues, and several advantages over conventional insulators have been noted [Harris2008]. However, no present operating accelerator uses this technology due to a lack of understanding of the HGI's performance in an operational environment. Another application area is in the renewed development of explosively driven magnetic flux compression generators [Knoepfel1970 ]. This development has engendered an interest in insulators exposed to longer pulses (several microseconds), ultraviolet radiation, high magnetic fields, and both vacuum and gas interfaces. Scaled testing [Javedani2007, Houck2006, Houck2008a] is ongoing, although it is not possible to duplicate the operational environment in the laboratory.

The Plasma Theory and Simulation Group at the University of California at Berkeley (UCB) has performed substantial research on dielectric breakdown and surface emission in the context of high power microwave devices, with over 40 publications over the last 3 years. Geometric effects on field emission, including means of reducing the order of the spatial model and requirements for resolving fine features with the mesh using an effective geometric field enhancement factor were developed [Feng2005]. The transition from Fowler Nordheim field emission at low surface electric field to the Space Charge Limited Fowler-Nordheim (SCL-FN) behavior was first characterized at Berkeley [Feng2006, Feng2008a] and extended to the relativistic regime [Feng2008b]. Studies of two-surface multipactors between electrodes demonstrated the roles of space charge and cavity detuning in achieving saturation [Gopinath1998]. The space charge shielding effects in single surface multipactor were investigated using XOOPIC [Valfells2000b]. New time-dependent effects were discovered in single surface microwave driven dielectric multipactors [Kim2005]. The study of the transition of microwave breakdown at dielectric windows from single surface multipactor at low pressure to collisional discharge at high pressure followed [Kim2006, Kim2007a, Verboncoeur2006]. The effects of wave propagation through non-uniform plasma slabs were also considered [Kim2007b]. A general scaling law for the breakdown time for different background gases over a wide range of pressures was developed [Lau2006]. Discrepancies in analytic, fluid, and kinetic particle models were considered [Kim2007c]. Generation of xrays in particle-surface impact events was characterized as a potential source of electrons for breakdown [Lim2007]. A novel global model with an enhanced EEDF was developed to study collisional breakdown [Nam2008a], used to demonstrate the frequency effects [Nam2008b], and extended to electronegative gases and air [Nam2008c].

Calabazas Creek Research, Inc. (CCR), in collaboration with the Plasma Theory and Simulation Group at the University of California - Berkeley, investigated models based on existing particle-in-cell, global models, and hybrid fluid models for analyzing surface breakdown of dielectric insulators and incorporate these into CCR's Visual Research Laboratory (VRL). The Visual Research Laboratory is a computer-based laboratory for performing experiments with high-voltage, high-current, electron-driven sources and applications of electromagnetic radiation. The applications range from fundamental physics in the high-voltage, high-current environments, to high power microwave sources 
for directed energy weapons, nuclear effects simulation, and novel imaging concepts for medical or materials industries.

\subsection{Phase I Results}

As discussed in detail for each task below, the Phase I project has demonstrated the feasibility of a modeling approach to analyzing breakdown effects, and for designing improved high voltage insulating systems. Development of the fundamental tools and models, and applications to classical DC high voltage insulator breakdown problems, provides a strong foundation for pursuing advanced model development and physics understanding in Phase II.

\subsubsection{Task 1: Angled dielectric insulator breakdown}

In modeling the breakdown of classical, angled-dielectric, triple-point insulators, a rotated geometry is used to properly account for particle orbits at the dielectric boundary as in Figure 3.

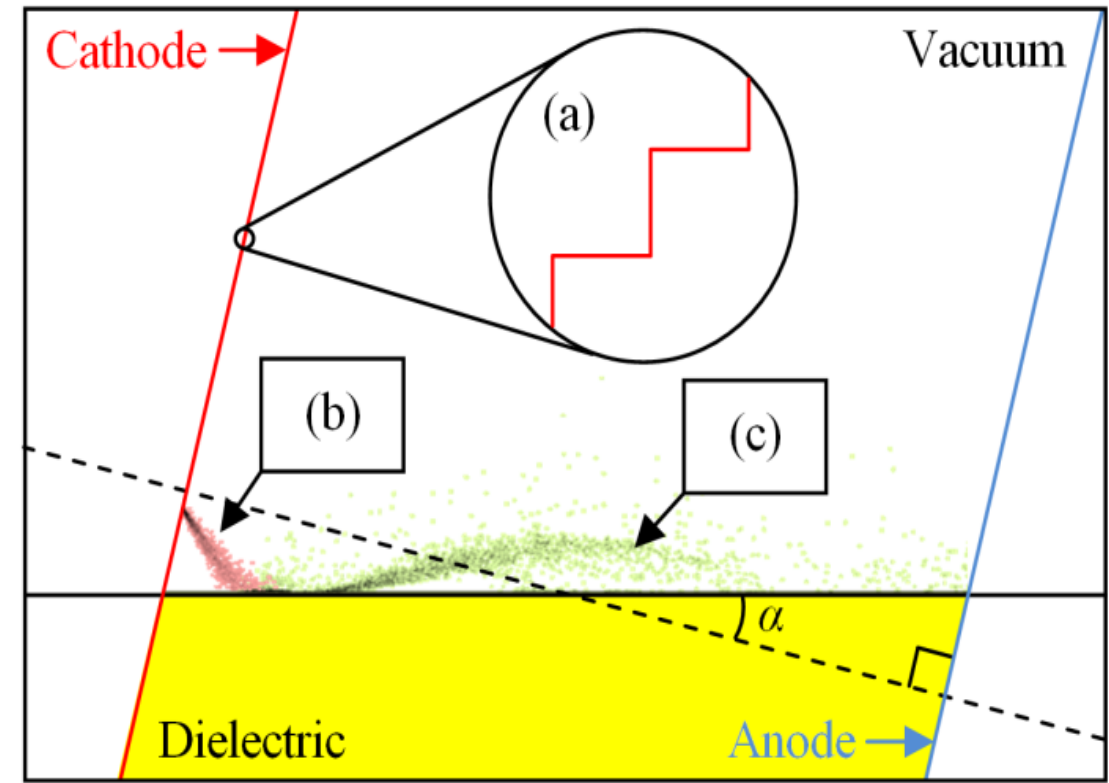

Figure 3. Phase space for primaries on Teflon dielectric with Fowler-Nordheim source. Typical characteristics are: (a) stair-stepping of angled boundaries, (b) typical primary-electron phase space, and (c) typical secondary-electron phase space. $\alpha$ is shown with respect to the dielectric.

One of the key mechanisms of dielectric breakdown is secondary-electron emission due to primary-electron impact on the insulator. This process is sensitive to impact angle and energy, and a discretized spatial domain can introduce surprisingly significant errors in the secondary-emission process at the dielectric. Grid errors are typical of the stairstepping in Figure 3(a). The rotated geometry eliminates stair-step grid errors on the dielectric, allowing correct resolution of the electron impact position, angle and energy. Furthermore, the upper and lower boundaries of Figure 3 must be placed many times the gap width away from the insulator surface in order to minimize their influence on the fields at the surface; however, it was demonstrated in Phase I that the influence can be 
minimized by employing a boundary with a linear voltage variation, which matches the boundary condition at infinity, as close as one gap width from the insulator surface.

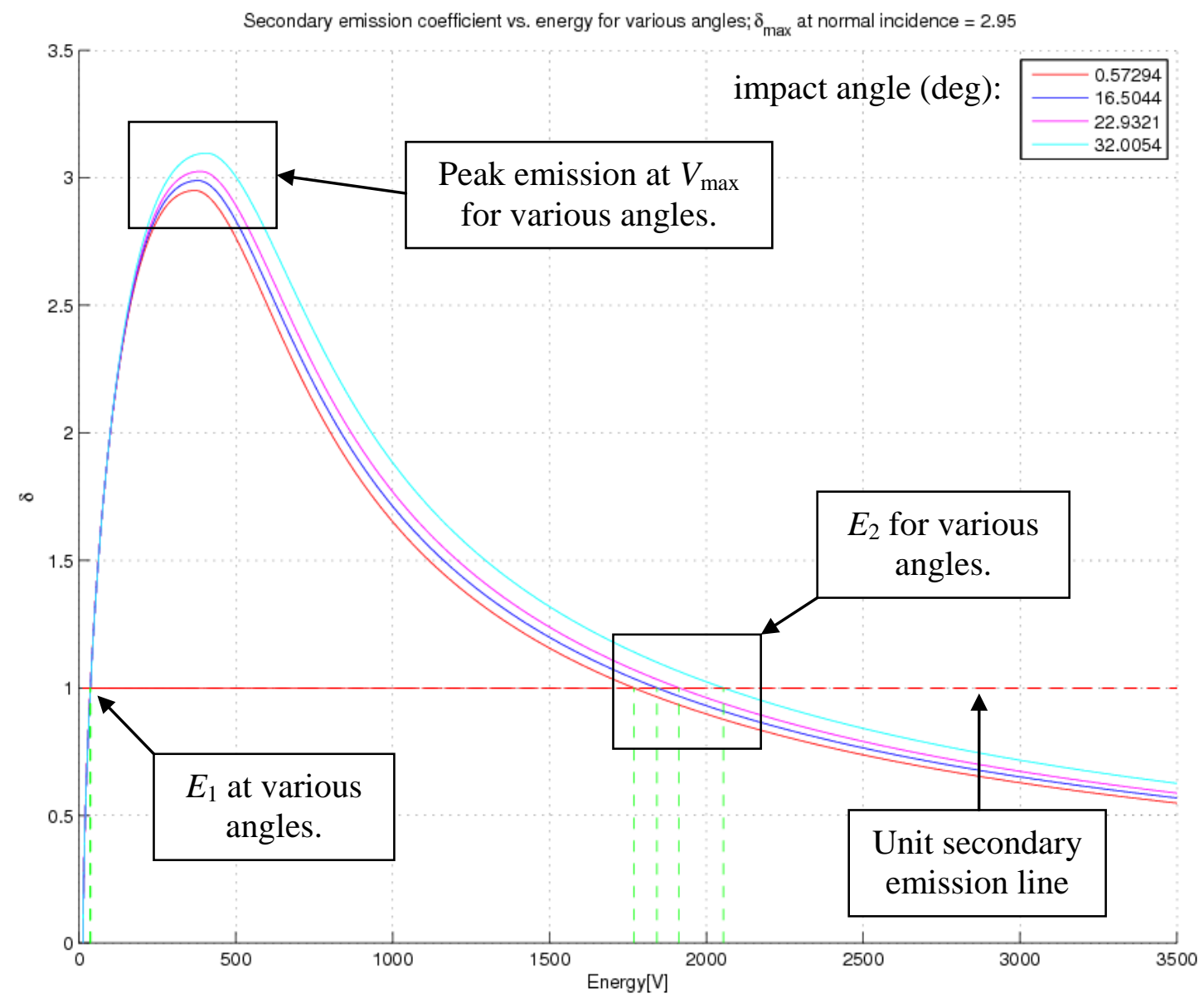

Figure 4. Vaughan model for secondary emission matched to Baker data for Teflon.

The secondary emission due to multipactor effects is modeled using the energy and angular dependent Vaughan model [Verboncoeur2005]. This model was improved to include data for the energy dependence of the secondary emission yield of teflon using the Baker data [need reference], as shown in Figure 4. The secondary emission yield is parameterized as follows:

$$
\delta(\mathrm{E}, \theta)=\delta_{\max 0}\left(1+k_{s d} \frac{\theta^{2}}{2 \pi}\right) W(w)
$$

where

$$
W(w)=\left\{\begin{array}{c}
(w \exp (1-w))^{k}, w \leq 3.6 \\
1.125 w^{-0.35}, w>3.6
\end{array},\right.
$$




$$
\begin{gathered}
w=\frac{\mathrm{E}-\mathrm{E}_{0}}{\mathrm{E}_{\max 0}\left(1+k_{s w} \theta^{2} / 2 \pi\right)-\mathrm{E}_{0}}, \\
k=\left\{\begin{array}{c}
0.56, w<1 \\
0.25,1 \leq w \leq 3.6
\end{array},\right.
\end{gathered}
$$

where $E$ is the impact energy, $E_{0}$ is the secondary emission threshold energy, $E_{\max 0}$ is the energy at which the peak, $\delta_{\max 0}$ in the secondary emission yield occurs, $\theta$ is the angle of impact with respect to the normal, and $0<k_{s d}, k_{s w}<2$ are empirical surface roughness coefficients, where the higher value indicates a highly polished surface. The model allows emission of true secondaries with an isotropic Maxwell-Boltzmann distribution, as well as a smaller percentage of scattered and reflected primaries. The shape is universal for many materials, where the primary differences are the location and amplitude of the maximum, as well as the location of the second crossover energy, $E_{2}$.

An electron source is assumed to exist near the triple-point junction between vacuum, dielectric, and metal, illustrated in Figure 3(b). Initially, a constant electron source was specified to investigate the general behavior of the system. This source model assumes a fixed source of electrons, which is unlikely in real systems, but provides pertinent insight into the interplay of the secondary emission and dielectric surface charge. The temporal accumulation of charge along the dielectric surface is shown in Figure 5. 


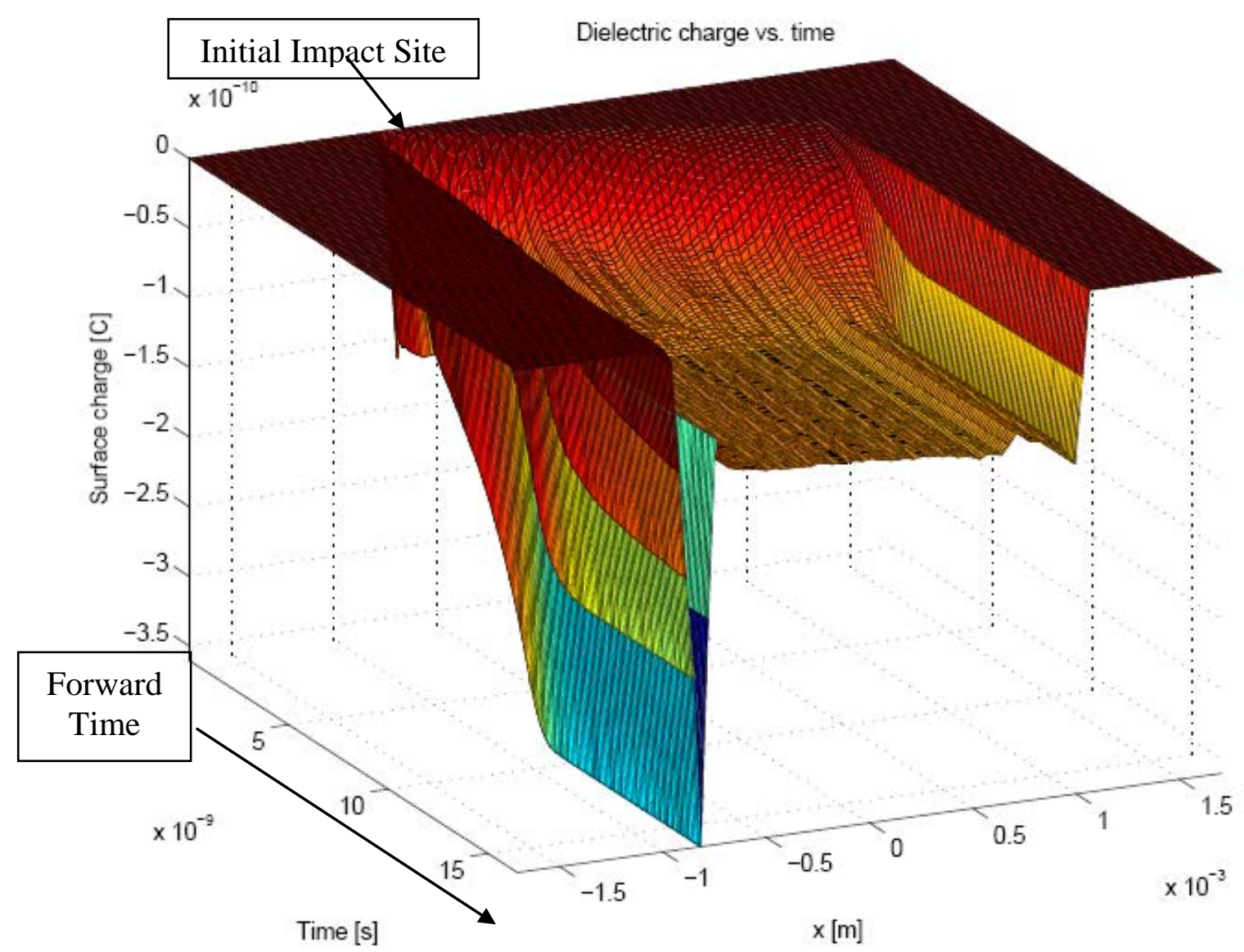

Figure 5. Dielectric surface charge over time for a constant electron source for Teflon dielectric in a $2 \mathrm{~mm}$ gap driven by $500 \mathrm{~V}$. The cathode intersects the dielectric at $-1 \mathrm{~mm}$, and the anode intersects at $1 \mathrm{~mm}$.

In Figure 5, a source with a thin spatial profile is specified, leading to a narrow impact profile. In cases with typical secondary-emission parameters, the dielectric surface charge usually builds negative for all time due to the low emission energy of the true secondaries coupled with the local attractive force that pulls the low-energy secondaries back to the dielectric near the impact site, reducing the effective secondary electron emission coefficient. When the surface charge is sufficient, secondaries can traverse larger distances across the dielectric, gaining energy in the electric field along the way. When secondaries are generated, a relatively positive charge is left behind in the dielectric, resulting in a local electric field that pulls electrons back to the surface. Secondaries that eventually return to the dielectric after a sufficient excursion can have enough impact energy to generate more secondaries. The primary-electron energy distribution can be observed through the simulation, showing initial impact energies above the first crossover energy, $E_{1}$, but below the second crossover energy, $E_{2}$. Figure 6 shows a typical primary-electron energy distribution after secondary-emission quenching. 


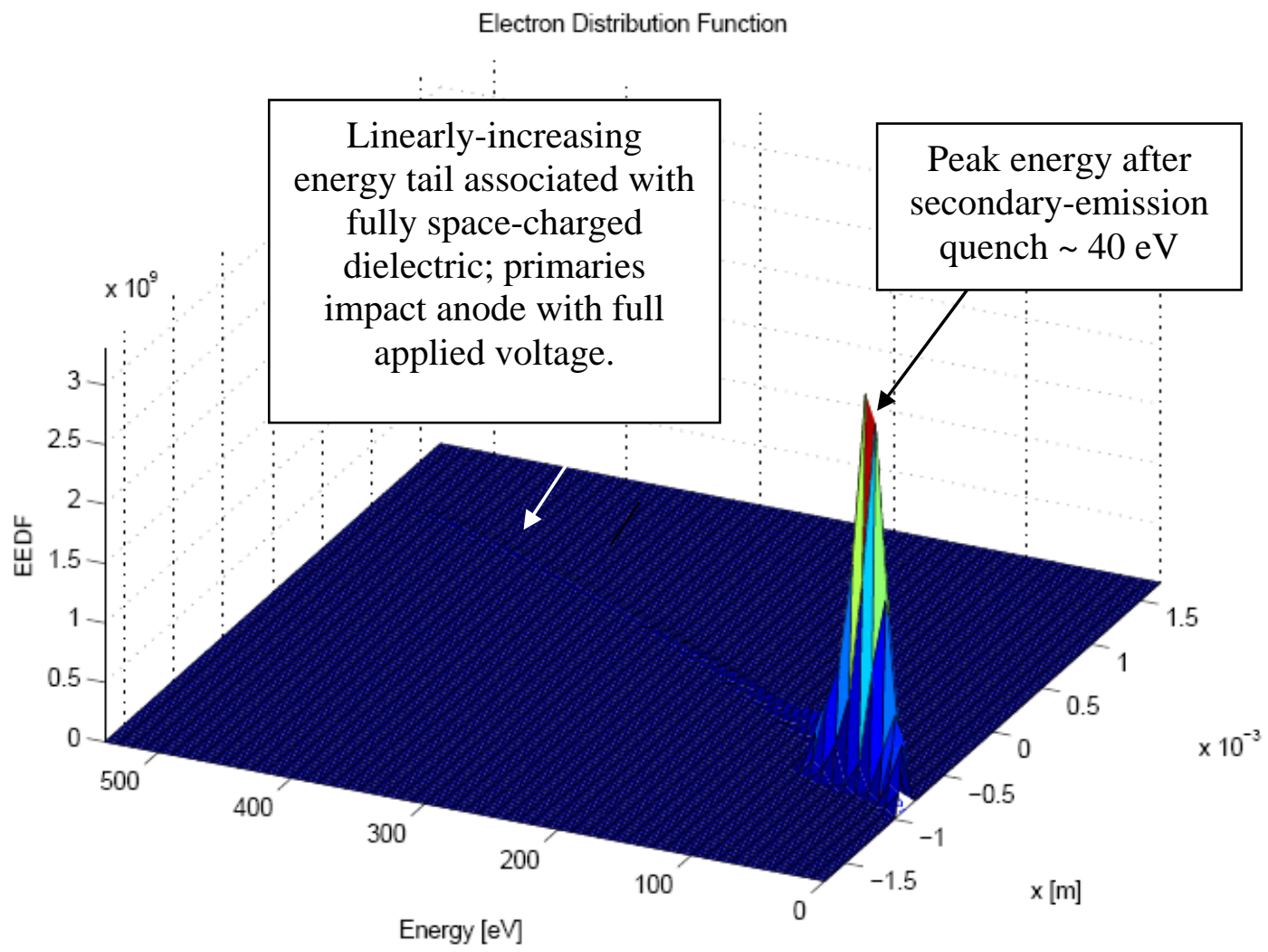

Figure 6. Primary-electron energy distribution (cumulative number of electrons) impacting the insulator surface. Secondary emission quenched.

Before the profile of Figure 6 is reached, the secondary-emission can avalanche from the dielectric to the anode, resulting in a measured current at the anode. This is shown in Figure 7. A current develops at the anode comprised entirely of secondaries until $5 \mathrm{~ns}$. A little before $5 \mathrm{~ns}$, the system space and surface charge has become significant enough to deflect primaries from the cathode to the anode. The increase in the primary current is a result of the sudden deflection of nearly the entire source current. Some current still makes its way to the dielectric, but primary impact energies have moved below the first crossover, and no further secondaries are measured. 


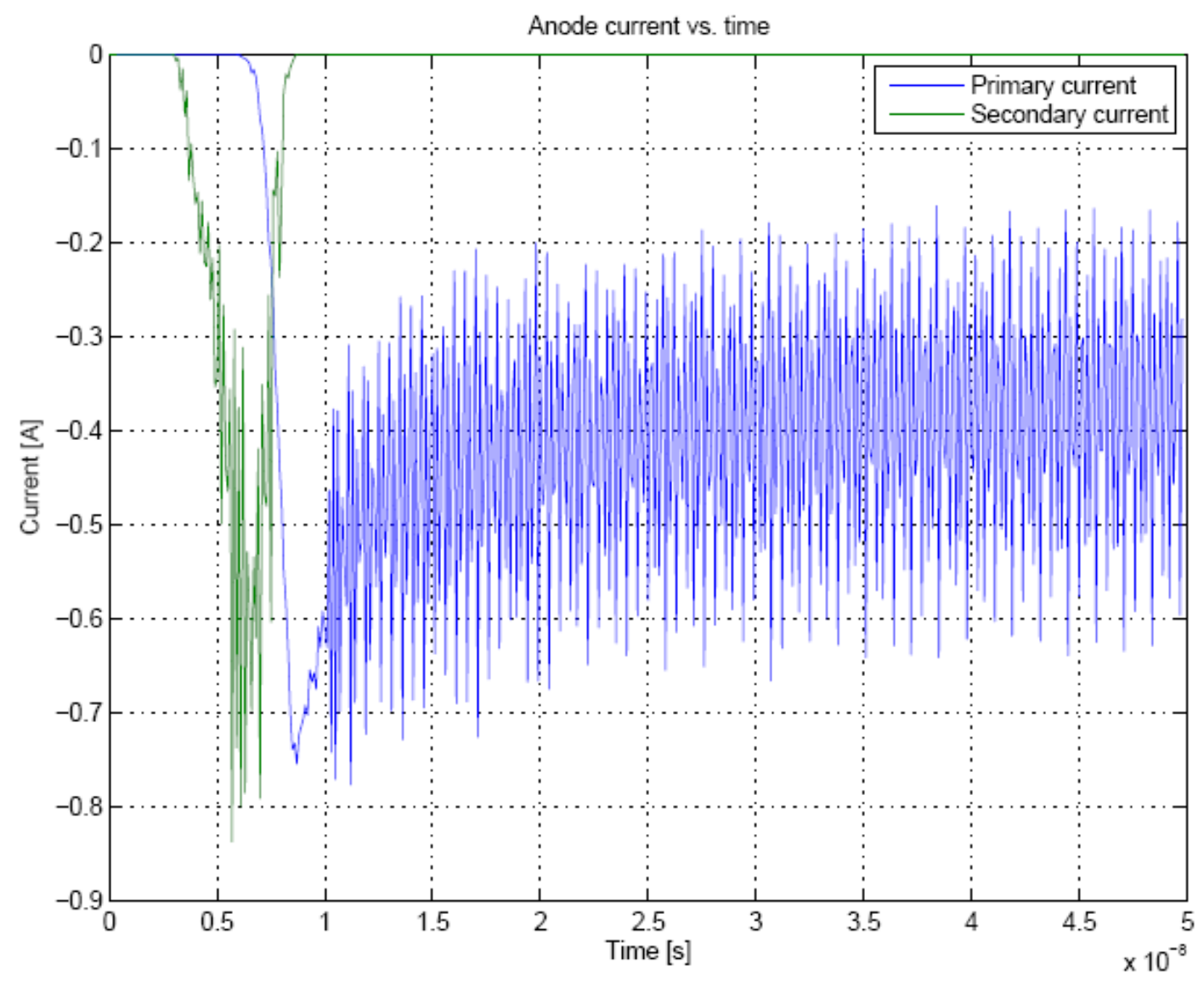

Figure 7. Primary-electron and secondary-electron current at the anode over time.

Next, the model is modified to include the injection current feedback of a FowlerNordheim (FN) emitter. The dielectric charge of an FN model is shown in Figure 6. Similar characteristics are seen for a self-consistent FN emitter comparing to Figure 2, but the surface charge is more distributed at the onset. 


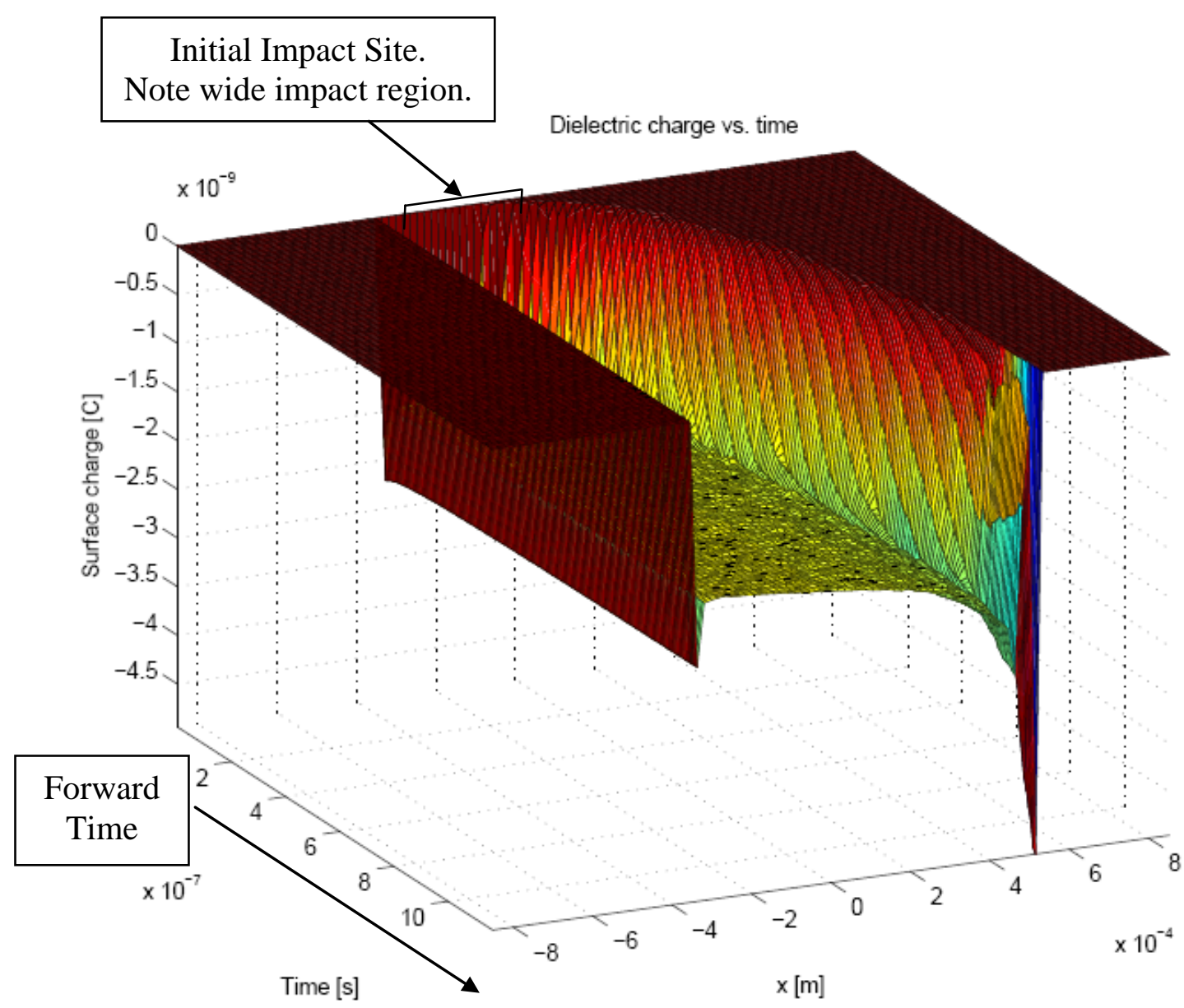

Figure 8. Dielectric surface charge over time for a self-consistent Fowler-Nordheim electron source in a 1 mm gap with $500 \mathrm{~V}$ applied. The cathode intersects the dielectric at $-0.5 \mathrm{~mm}$, and the anode intersects at $0.5 \mathrm{~mm}$.

The FN emitter is seeded with a field-enhancement factor to simulate a small surface feature, producing an appreciable number of electrons in the initial fields. This might be achieved in real systems by piercing a needle through the cathode surface, or roughening the surface. If the insulator surface charges positively, the local field at the FN emitter will be enhanced and the emission will increase, as will the primary impact energy on the dielectric, causing further positive charging. This process should saturate when the surface charge is sufficient to cause the primaries to impact at the second cross over energy, $E_{2}$. However, if low energy secondaries are attracted back to the surface near the emission point, the effective secondary emission coefficient can be less than one, resulting in negative charging of the surface, and reducing the surface field at the FN emitter, and eventually deflecting the primaries away from the insulator surface resulting in the wide distribution of negative charge shown in Figure 8. 


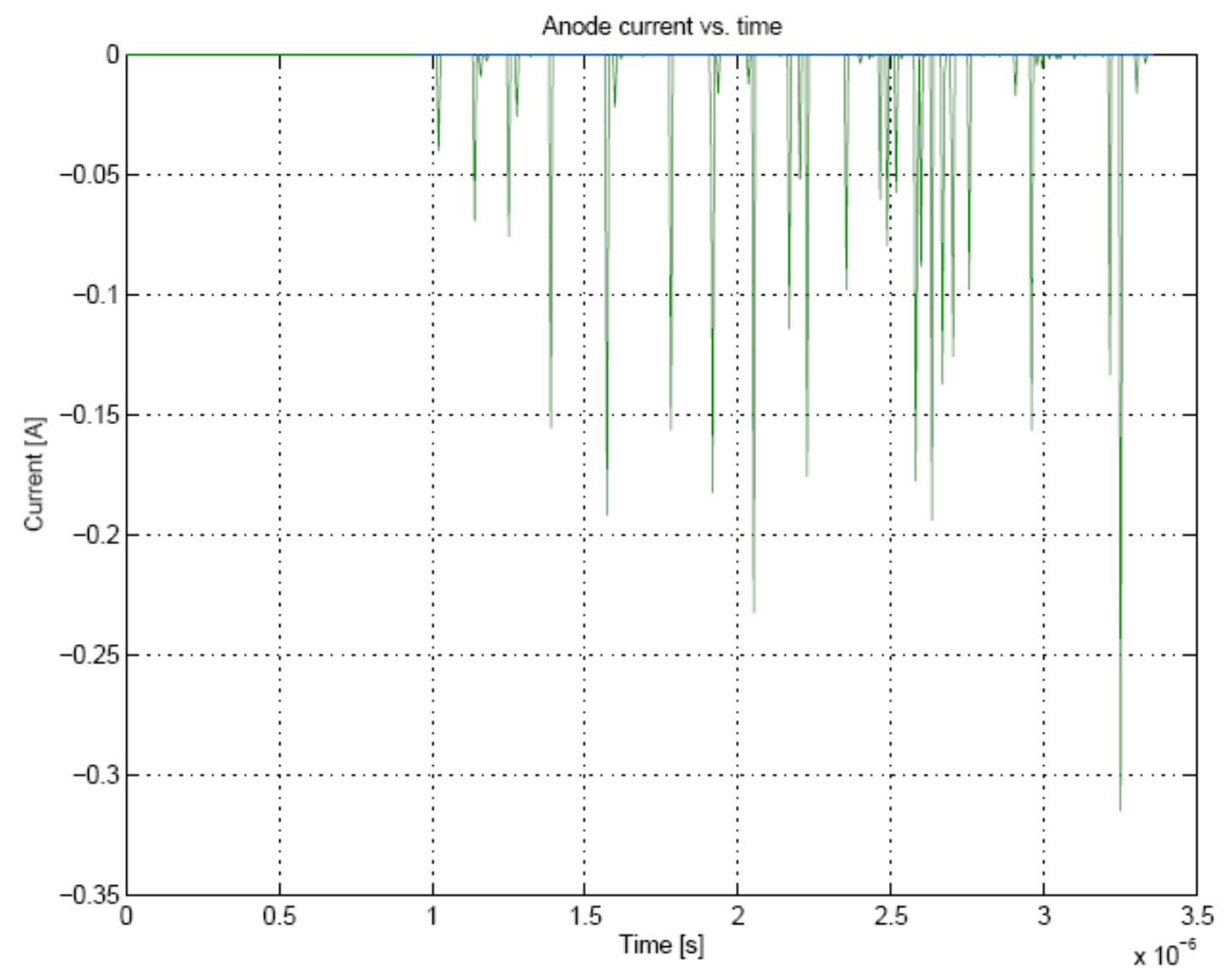

Figure 9. Secondary current at the anode vs. time for Fowler-Nordheim source model.

The current profile corresponding to the negative charging scenario is shown in Figure 9, showing an intermittent secondary current on the anode. No primaries have reached the anode in this case, illustrating the importance of the space-charge profile in reaching any sort of breakdown scenario. The current is reduced in the FN case with the wider initial-charge profile. This case better represents real systems with seed-electron sources that depend on the electric-field strength and system characteristics.

It is clear from these Phase I results that the mechanics of modeling a single gap vacuum insulator are feasible. The tools are now in place to proceed.

\subsubsection{Task 2: Stack of angled insulators}

We model the dielectric insulator stack in the same way that we model the monolithic insulator, introducing additional electrodes with intermediate applied potential. The general model is shown in Figure 10. Note that the center electrode is shown in a closeup view, but only extends $1 \mathrm{~mm}$ above the insulator. 


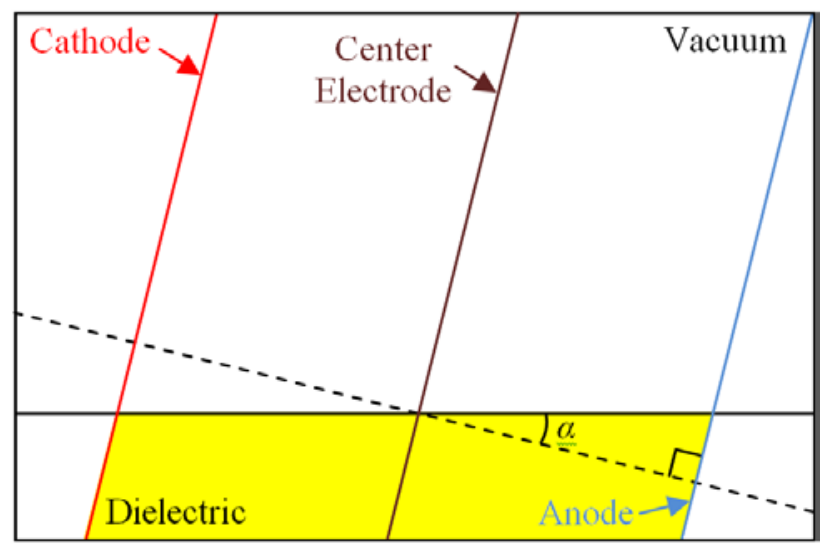

Figure 10. General stacked-electrode model.

A proof-of-principle calculation is provided. The resulting dielectric charge is shown in Figure 11.

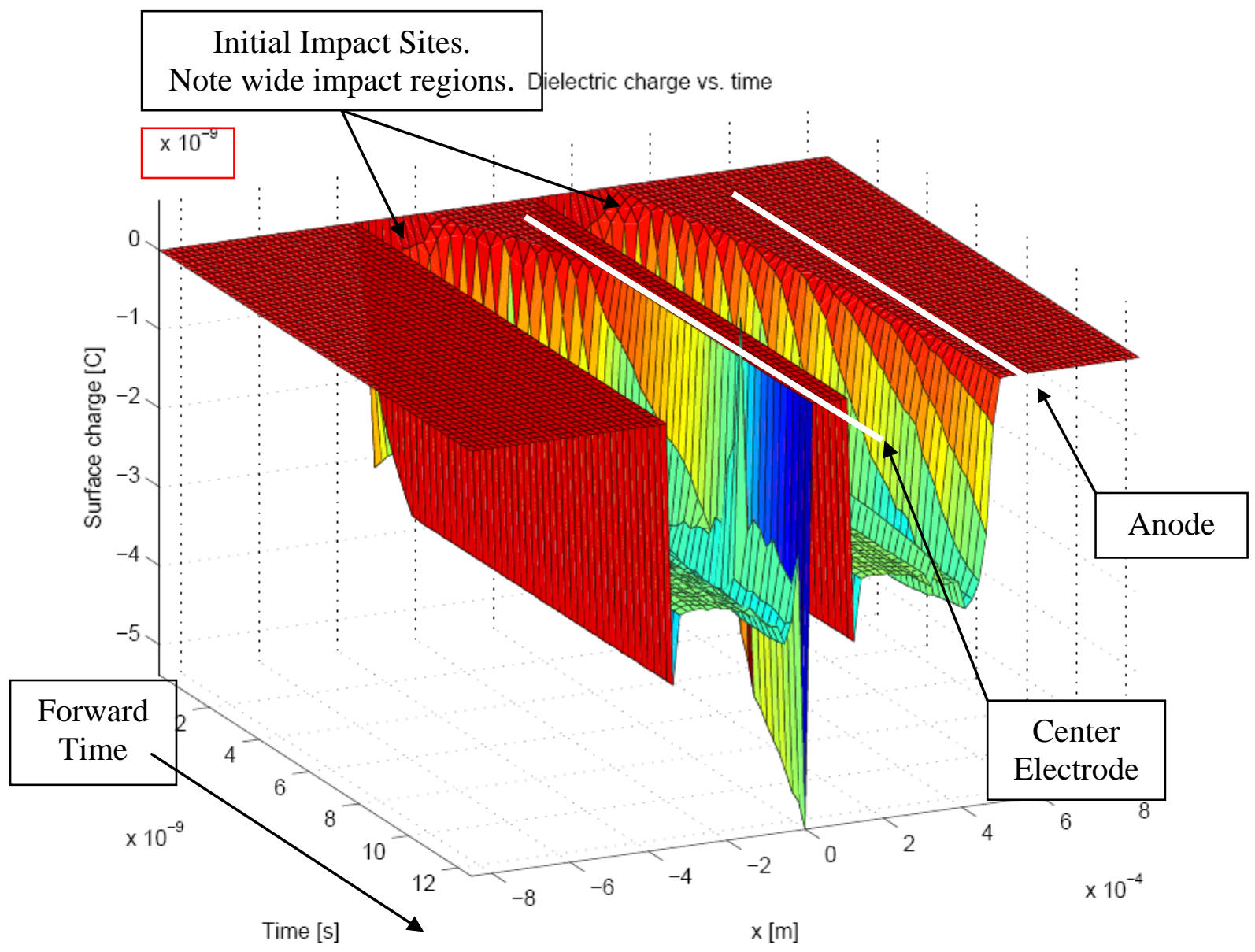

Figure 11. Surface charge for stacked dielectric insulation. Center electrode placed at intermediate potential. Fowler-Nordheim parameters chosen so both cathode and center electrode are in field-emission regimes.

The same characteristics can be seen as in the monolithic case. Note that this case is not necessarily typical since current sources are assumed at both electrodes. FN sources 
are sensitive to the local field and surface characteristics. A system at the onset of FN emission at the cathode can be modeled such that no FN emission occurs at the center unless further surface charging occurs near the center electrode.

The feasibility of modeling a stacked cell insulator was successfully demonstrated in Phase I, again with the need for further investigation of the physics.

\subsubsection{Task 3: Advanced models}

In Task 3, a number of advanced models were considered.

The boundary conditions at the open ends of the gap (top and bottom in Figure 3) pose a challenge. The physical ends of the gap are located at a distance from the insulator surface which is very large compared to the gap width. Since all the physics takes place within less than $1 \mathrm{~mm}$ from the surface for most configurations, the challenge is to use a boundary condition which does not influence the electric fields in the region of interest, while keeping the simulated region small for efficiency. In Phase I, we found that setting the derivative of the potential to zero normal to the boundary was inadequate, and influenced the results even at relatively large distances. However, setting the potential to a fixed value varying linearly from cathode to anode voltage worked very well, and allowed reduction of the system size to be about one gap width normal to the insulator. This has been implemented and tested in Phase I.

In the course of progressing to a self-consistent breakdown model, the field emission plays a crucial role early in the process. Hence, it will be important to improve the fidelity of the fields at the electrode surfaces. Indeed, it can be shown that for stairstepped structures, the field at the covex corners approaches infinity as the cell size approaches zero, and the field at the concave corners approaches zero as the cell size approaches zero. Hence, it is determined that a piecewise continuous boundary condition will be important in Phase II. A classical solution to this problem is to replace a single point with a block of points, and solving for difference equation by extrapolating the solution of trial functions [Shortley1938].

In order to model a breakdown event self-consistently, desorption due to localized electron heating must be included. A model of surface desorption of lattice impurities, based on electronic sputtering, shows a strong dependence of yield $\mathrm{Y}$ on angle of incidence $\theta$ [Molvik2004]:

$$
\frac{Y(\theta)}{Y_{0}}=1+1.82 \times 10^{-4} \exp (0.09 \times \theta),
$$

where $\theta$ is the angle with respect to the surface normal, and $Y_{0}$ is material-based yield at normal incidence. The energy distribution of emitted neutrals for binding energy $U$ is given by [Thompson1968]:

$$
f(E)=C_{1} \frac{2 U E}{E_{e x c}(E+U)^{3}} .
$$

where $E_{e x c} \approx d E / d x /\left(n \pi r^{2}\right)$ is the excitation energy [Berthold1997], $n$ is the surface atom density, $r$ is the radius of energy deposition, and $C_{1}$ is an arbitrary constant. Here, 
$d E / d x$ is the stopping power of the material. Inverting gives the cumulative distribution, from which we obtain the emitted energies of neutrals for a random number $0<R<1$ :

$$
\frac{E}{U}=\frac{R+R^{1 / 2}}{1-R} .
$$

The angular dependence is taken to be [Bringa1999]:

$$
f(\theta)=C_{2}(\cos \theta)^{2},
$$

where $C_{2}$ is an arbitrary constant.

In Phase I we found a high sensitivity to the characteristics of the true secondaries, including their emitted distribution. Since the slow electrons are susceptible to failing to escape the local surface charge, and their impact energy is generally well below the first cross over energy of the secondary emission curve, the distribution is crucial in determining the effective secondary yield in the presence of an accelerating field. Hence obtaining an improved yield for the materials employed in Phase II will of prime importance.

In summary, the advanced model elements of Task 3 will comprise key elements of a quatitative Phase II effort to model breakdown physics. These models generally involve minor modifications of XOOPIC and VRL, so implementing them in a Phase II program is feasible and will result in improved fidelity. Only the piecewise continuous boundary elements will involve significant complexity

\subsubsection{Task 4: VRL integration}

The models implemented in Phase I were successfully incorporated into the VRL code due to its flexible design.

\section{Summary}

The Phase I program demonstrated the feasibility of developing an advanced code for modeling insulator breakdown. Such a code would be of great interest for a number of applications, including high energy physics, microwave source development, fusion sciences, and other research and industrial applications using high voltage devices.

\section{Bibliography \& References Cited}

1. [Ang1998] L.-K. Ang, Y. Y. Lau, R. A. Kishek, and R. M. Gilgenbach, "Power deposited on a dielectric by multipactor", IEEE Trans. Plasma Sci. 26, 290-295 (1998).

2. [Berthold1997] W. Berthold and A. Wucher, "Energy- and Angle-Dependent Excitation Probability of Sputtered Metastable Silver Atoms”, Physical Rev. B 56, 4251-4260 (1997).

3. [Bringa1999] E. M. Bringa, R. E. Johnson, and L. Dutkiewicz, "Molecular dynamics study of non-equilibrium energy transport from a cylindrical track: Part II Spike models for sputtering yield”, Nucl. Instr. Meth. in Phys. Res. B152, 267-290 (1999).

4. [Elizondo1992] J.M. Elizondo and A.E. Rodriquez, "Novel High Voltage Vacuum 
Surface Flashover Insulator Technology,” Proc. $15^{\text {th }}$ Int. Symp. Discharges and Electrical Insulation in Vacuum (Vde-Verlag Gmbh, Berlin), 198-202 (1992)

5. [Feng2005] Y. Feng and J. P. Verboncoeur, "A model for effective field enhancement for Fowler--Nordheim field emission", Phys. Plasmas 12, 10330113306 (2005).

6. [Feng2006] Y. Feng and J. P. Verboncoeur, "Transition from Fowler-Nordheim field emission to space charge limited current density," Phys. Plasmas 13, 073105-8 (2006).

7. [Feng2008a] Y. Feng, J. P. Verboncoeur, and M.-C. Lin, "Solution for space charge limited field emission current densities with injection velocity and geometrical effects correction", Phys. Plasmas 15, 043301 (2008).

8. [Feng2008b] Y. Feng and J. P. Verboncoeur, "Consistent solution for space-chargelimited current in the relativistic regime for mono-energetic initial velocities", accepted Phys. Plasmas (2008).

9. [Gopinath1998] V. P. Gopinath, J. P. Verboncoeur and C. K. Birdsall, "Multipactor electron discharge physics using an improved secondary emission model”, Phys. Plasmas 5, 1535-1540 (1998).

10. [Harris2008] J.R. Harris et al., "Vacuum insulator development for the dielectric wall accelerator,” J. Appl. Phys. 104, 023301 (2008)

11. [Houck1997] T.L. Houck et al., "Stacked Insulator Induction Accelerator Gaps," UCRL-JC-127273 May 1997, 1997 Particle Accelerator Conference May 1997

12. [Houck2006] T.L. Houck, D.A. Goerz, J.B. Javedani, E.J. Lauer, L.K. Tully, G.E. Vogtlin, "Study of Vacuum Insulator Flashover for Pulse Lengths of MultiMicroseconds,” UCRL-CONF-223361 31 July 2006, Presented at the 2006 Linear Accelerator Conference August 2006

13. [Houck2008a] T.L. Houck, J.B. Javedani, L.K. Tully, G.E. Vogtlin, R. Speer, A. Ferriera, "Evaluation of Magnetic Insulation in SF6 Filled Regions," LLNL in progress Strategic Mission Research Project \#SM-08-048

14. [Houck2008b] T. L. Houck, private communication (2008).

15. [Javedani2007] J.B. Javedani, D.A. Goerz, T.L. Houck, E.J. Lauer, R.D. Speer, L.K.Tully, G.E. Vogtlin, A.D. White, "Understanding and Improving High Voltage Vacuum Insulators for Microsecond Pulses,” UCRL-TR-228713 06 March 2007

16. [Jordan2007] N.M. Jordan, Y.Y. Lau, D.M. French, R.M. Gilgenbach, and P. Pengvanich, "Electric field and electron orbits near a tripl point”, J. Appl. Phys. 102, 033301-033310 (2007).

17. [Kim2005] H. C. Kim and J. P. Verboncoeur, "Time-dependent physics of a singlesurface multipactor discharge", Phys. Plasmas 12, 123504-123507 (2005).

18. [Kim2006] H. C. Kim and J. P. Verboncoeur, "Transition of window breakdown from vacuum multipactor discharge to rf plasma", Phys. Plasmas 13, 123506-6 (2006).

19. [Kim2007a] H. C. Kim, J. P. Verboncoeur, and Y. Y. Lau, "Modeling RF window breakdown: from vacuum multipactor to RF plasmas", IEEE-Trans. Dielec. Elec. Insul. 14, 766-773 (2007) invited.

20. [Kim2007b] H. C. Kim and J. P. Verboncoeur, "Reflection, absorption, and 
transmission of $\mathrm{TE}_{\mathrm{m}, 0}$ electromagnetic waves propagating in a nonuniform plasma slab", Comput. Phys. Comm. 177, 118-121 (2007) invited

21. [Kim2007c] H.-C. Kim and J. P. Verboncoeur, "Validity of Two-Term Boltzmann Approximation Employed in Fluid Models", 34th IEEE ICOPS, Albuquerque, NM (2007)

22. [Kishek1998] R. A. Kishek and Y. Y. Lau, "Multipactor discharge on a dielectric", Phys. Rev. Lett. 80, 193-196 (1998).

23. [Knoepfel970] H. Knoepfel, Pulsed High Magnetic Fields, American Elsevier Publishing Co., NY 1970 Chapters 8 and 9

24. [Lau2006] Y. Y. Lau, J. P. Verboncoeur, and H. C. Kim, "Scaling laws for dielectric window breakdown in vacuum and collisional regimes", Appl. Phys. Lett. 89, 261501-3 (2006).

25. [Lim2007] C.-H. Lim and J. P. Verboncoeur. "Modeling X-Ray Emission in a High Voltage Vacuum Gap Including Secondary Electron Emission", 34th IEEE ICOPS, Albuquerque, NM (2007)

26. [Molvik2004] A. Molvik, curve fit based on SRIM data, private communication (2004).

27. [Nam2008a] S. K. Nam and J. P. Verboncoeur, "Effect of Electron Energy Distribution Function on the Global Model for High Power Microwave Breakdown at High Pressures", Appl. Phys. Lett. 23, 231502:1-3 (2008).

28. [Nam2008b] S. K. Nam and J. P. Verboncoeur, "Effect of Microwave Frequency on Breakdown and Electron Energy Distribution Function using a Global Model", Appl. Phys. Lett. 93, 151504-6 (2008).

29. [Nam2008c] S. K. Nam and J. P. Verboncoeur, "Global model for high power microwave breakdown at high pressure in air", accepted for publication in Comp. Phys. Comm. (2008).

30. [Sampayan1995] S. Sampayan, et. al., "High Gradient Insulator Technology for the Dielectric Wall Accelerator ," Proceedings of the 1995 Particle Accelerator Conference, (IEEE), New York, N.Y., pp.1269-1271 (1995)

31. [Scarpetti1997] R.D. Scarpetti, et al., "Upgrades to the LLNL Flash X-Ray Induction Linear Accelerator (FXR)," UCRLFJG12S3 30 June 1997, Presented at the $11^{\text {th }}$ IEEE International Pulsed Power Conference (1997)

32. [Shortley1938] G. H. Shortley and R. Weller, “The Numerical Solution of Laplace’s Equation”, J. Applied Phys. 9, 334-348 (1938).

33. [Thompson1968] M. W. Thompson, Philosophical Magazine 18, 377 (1968)

34. [Valfells2000a] A. Valfells, L. K. Ang, Y. Y. Lau, and R. M. Gilgenbach, "Effects of an external magnetic field, and of oblique radio-frequency electric fields on multipactor discharge on a dielectric”, Phys. Plasmas 7, 750-757 (2000).

35. [Valfells2000b] A. Valfells, J. P. Verboncoeur, and Y. Y. Lau, "Space-charge effects on multipactor on a dielectric”, IEEE Trans. Plasma Sci. 28, 529-536 (2000).

36. [Verboncoeur1993] J. P. Verboncoeur, M. V. Alves, V. Vahedi and C. K. Birdsall, "Simultaneous Potential and Circuit Solution for 1D Bounded Plasma Particle Simulation Codes", J. Comput. Phys. 104 (1993). 
37. [Verboncoeur1995] J. P. Verboncoeur, A. B. Langdon and N. T. Gladd, "An ObjectOriented Electromagnetic PIC Code", Comput. Phys. Comm. 87, 199-211 (1995).

38. [Verboncoeur2005] J. P. Verboncoeur, "Particle simulation of plasmas: review and advances", Plasma Physics and Controlled Fusion 47, A231-A260 (2005), invited.

39. [Verboncoeur2006] J. P. Verboncoeur, H. C. Kim, Y. Chen, and Y. Y. Lau, "Modeling RF Window Breakdown: From Vacuum Multipactor to Volumentric Ionization Discharge", Conf. Record 26th Int. Power Modulator Symposium and 2006 High-Voltage Workshop, Washington D.C., IEEE Cat. No. 06CH37746, 13-16 (2006), invited.

40. [Vogtlin1989] G. E. Vogtlin and J.E. Vernazza, "Vacuum Insulator Failure Measurements and Improvement," UCRL-100361 June 1989, Presented at the $7^{\text {th }}$ IEEE Pulsed Power Conference (1989)

41. [VRL2008] Computational Virtual Laboratory for High Voltage Radiation Experiments, U.S. Air Force contract FA9550-05-C-0081, August 2005 to January 2008. 